\title{
Grothendieck bialgebras, Partition lattices, and symmetric functions in noncommutative variables
}

\author{
N. Bergeron*1, C. Hohlweg*2, M. Rosas*1, and M. Zabrocki*1. \\ ${ }^{*}$ Department of Mathematics and Statistics, \\ York University \\ Toronto, Ontario M3J 1P3, Canada. \\ bergeron@mathstat.yorku.ca, mrosas@us.es, zabrocki@mathstat.yorku.ca \\ ${ }^{* 2}$ The Fields Institute \\ 222 College Street \\ Toronto, Ontario, M5T 3J1, Canada. \\ chohlweg@fields.utoronto.ca
}

Submitted: Jul 14, 2005; Accepted: Jul 19, 2006; Published: Aug 25, 2006

Mathematics Subject Classifications: 05E05, 05E10, 16G10, $20 \mathrm{C} 08$.

\begin{abstract}
We show that the Grothendieck bialgebra of the semi-tower of partition lattice algebras is isomorphic to the graded dual of the bialgebra of symmetric functions in noncommutative variables. In particular this isomorphism singles out a canonical new basis of the symmetric functions in noncommutative variables which would be an analogue of the Schur function basis for this bialgebra.
\end{abstract}

\section{Introduction}

Combinatorial Hopf algebras are graded connected Hopf algebras equipped with a multiplicative linear functional $\zeta: \mathcal{H} \rightarrow \mathbb{k}$ called a character (see [1]). Here we assume that $\mathbb{k}$ is a field of characteristic zero. There has been renewed interest in these spaces in recent papers (see for example $[3,4,6,11,13]$ and the references therein). One particularly interesting aspect of recent work has been to realize a given combinatorial Hopf algebra as the Grothendieck Hopf algebra of a tower of algebras.

The prototypical example is the Hopf algebra of symmetric functions viewed, via the Frobenius characteristic map, as the Grothendieck Hopf algebras of the modules of all

${ }^{*}$ This work is supported in part by CRC and NSERC. It is the results of a working seminar at Fields Institute with the active participation of T. MacHenry, M. Mishna, H. Li and L. Sabourin

THE ELECTRONIC JOURNAL OF COMBINATORICS 13 (2006), \#R75 
symmetric group algebras $\mathbb{k} S_{n}$ for $n \geq 0$. The multiplication is given via induction from $\mathbb{k} S_{n} \otimes \mathbb{k} S_{m}$ to $\mathbb{k} S_{n+m}$ and the comultiplication is the sum over $r$ of the restriction from $\mathbb{k} S_{n}$ to $\mathbb{k} S_{r} \otimes \mathbb{k} S_{n-r}$. The tensor product of modules defines a third operation on symmetric functions usually referred to as the internal multiplication or the Kronecker product $[16,22]$. The Schur symmetric functions are then canonically defined as the Frobenius image of the simple modules.

There are many more examples of this kind of connection (see $[5,12,15]$ ). Here we are interested in the bialgebra structure of the symmetric functions in noncommutative variables $[7,8,9,17,21]$ and the goal of this paper is to realize it as the Grothendieck bialgebra of the modules of the partition lattice algebras.

We denote by NCSym $=\bigoplus_{d \geq 0} \mathrm{NCSym}_{d}$ the algebra of symmetric functions in noncommutative variables, the product is induced from the concatenation of words. This is a Hopf algebra equipped with an internal comultiplication. The space $\mathrm{NCSym}_{d}$ is the subspace of series in the noncommutative variables $x_{1}, x_{2}, \ldots$ with homogeneous degree $d$ that are invariants by any finite permutation of the variables. The algebra structure of NCSym was first introduced in [21] where it was shown to be a free noncommutative algebra. This algebra was used in [9] to study free powers of noncommutative rings. More recently, a series of new bases was given for this space, lifting some of the classical bases of (commutative) symmetric functions [17]. The Hopf algebra structure was uncovered in $[2,7,8]$ along with other fundamental algebraic and geometric structures.

The (external) comultiplication $\Delta: \mathrm{NCSym}_{d} \rightarrow \bigoplus \mathrm{NCSym}_{k} \otimes \mathrm{NCSym}_{d-k}$ is graded and gives rise to a structure of a graded Hopf algebra on NCSym. The algebra NCSym also has an internal comultiplication $\Delta^{\odot}: \mathrm{NCSym}_{d} \rightarrow \mathrm{NCSym}_{d} \otimes \mathrm{NCSym}_{d}$ which is not graded. The algebra NCSym with the comultiplication $\Delta^{\odot}$ is only a bialgebra (not graded) and is different from the previous graded Hopf structure.

After investigating the Hopf algebra structure of NCSym, it is natural to ask if there exists a tower of algebras $\left\{A_{n}\right\}_{n \geq 0}$ such that the Hopf algebra NCSym corresponds to the Grothendieck bialgebra (or Hopf) algebra of the $A_{n}$-modules. This was the 2004-2005 question for our algebraic combinatorics working seminar at Fields Institute where the research for this article was done.

Our answer involves the partition lattice algebras $\left(\mathbb{k} \Pi_{n}, \wedge\right)$ and $\left(\mathbb{k} \Pi_{n}, \vee\right)$ (as well as the Solomon-Tits algebras $[10,18,20])$. For each one, with finite modules we can define a tensor product of $\mathbb{k} \Pi_{n}$ modules and a restriction from $\mathbb{k} \Pi_{n}$ module to $\mathbb{k}_{k} \otimes \mathbb{k} \Pi_{n-k}$ modules. This allows us to place on $\bigoplus_{n} G_{0}\left(\mathbb{k}_{n}\right)$, the Grothendieck ring of the $\mathbb{k} \Pi_{n}$, a bialgebra structure (but not a Hopf algebra structure). We then define a bialgebra isomorphism $\bigoplus_{n} G_{0}\left(\mathbb{k}_{n}\right) \rightarrow \mathrm{NCSym}^{*}$. We call this map the Frobenius characteristic map of the partition lattice algebras. This singles out a unique canonical basis of NCSym (up to automorphism) corresponding to the simple modules of the $\mathbb{k} \Pi_{n}$.

Our paper is divided into 4 sections as follows. In section 1 we recall the definition and structure of NCSym. We then state our first theorem claiming the existence of a basis $\mathbf{x}$ of NCSym defined by certain algebraic properties. The proof of it will be postponed to section 4 . In section 2 we recall the definition and structure of the partition lattice algebras $\mathbb{k} \Pi_{n}$ with the product given by the lattice operation $\wedge$ and define their modules. 
We then introduce a structure of a semi-tower of algebras (i.e. we have a non-unital embedding $\rho_{n, m}: \mathbb{k} \Pi_{n} \otimes \mathbb{k} \Pi_{m} \rightarrow \mathbb{k} \Pi_{n+m}$ of algebras) on the partition lattice algebras and show that it induces a bialgebra structure on its Grothendieck ring. Our second theorem states that this Grothendieck bialgebra is dual to NCSym. The classes of simple modules correspond then to the basis $\mathbf{x}$. In view of the work of Brown [10] we remark that this can also be done with the semi-tower of Solomon-Tits algebras. In section 3 we build the same construction with the lattice algebras $\mathbb{k} \Pi_{n}$ with the product $\vee$. With this tower of algebras (i.e. $\rho_{n, m}$ is a unital morphism of algebras) we find that the Grothendieck bialgebra is again dual to NCSym, but this time the classes of simple modules correspond to the monomial basis of NCSym.

In section 4 we give the proof of our first theorem and show the basis canonically defined in section 2 corresponds to the simple modules of the $\mathbb{k} \Pi_{n}$. In light of the Frobenius characteristic of section 2, the basis can be interpreted as an analogue of the Schur functions for NCSym and providing an answer to an open question of [17].

\section{NCSym and the basis $\left\{\mathrm{x}_{A}\right\}$}

We recall the basic definition and structure of NCSym. Most of it can be found in $[7,8]$. A set partition $A$ of $m$ is a set of non-empty subsets $A_{1}, A_{2}, \ldots, A_{k} \subseteq[m]=\{1,2, \ldots, m\}$ such that $A_{i} \cap A_{j}=\emptyset$ for $i \neq j$ and $A_{1} \cup A_{2} \cup \cdots \cup A_{k}=[m]$. The subsets $A_{i}$ are called the parts of the set partition and the number of non-empty parts the length of $A$, denoted by $\ell(A)$. There is a natural mapping from set partitions to integer partitions given by $\lambda(A)=\left(\left|A_{1}\right|,\left|A_{2}\right|, \ldots,\left|A_{k}\right|\right)$, where the list is then sorted so that the integers are listed in weakly decreasing order to form a partition.

We shall use $\ell(\lambda)$ to refer to the length (the number of parts) of the partition and $|\lambda|$ is the size of the partition (the sum of the sizes of the parts), while $n_{i}(\lambda)$ shall refer to the number of parts of the partition of size $i$. We denote by $\Pi_{m}$ the set of set partitions of $m$. The number of set partitions is given by the Bell numbers. These can be defined by the recurrence $B_{0}=1$ and $B_{n}=\sum_{i=0}^{n-1}\left(\begin{array}{c}n-1 \\ i\end{array}\right) B_{i}$.

For a set $S=\left\{s_{1}, s_{2}, \ldots, s_{k}\right\}$ of integers $s_{i}$ and an integer $n$ we use the notation $S+n$ to represent the set $\left\{s_{1}+n, s_{2}+n, \ldots, s_{k}+n\right\}$. For $A \in \Pi_{m}$ and $B \in \Pi_{r}$ set partitions with parts $A_{i}, 1 \leq i \leq \ell(A)$ and $B_{i}, 1 \leq i \leq \ell(B)$ respectively, we set $A \mid B=\left\{A_{1}, A_{2}, \ldots, A_{\ell(A)}, B_{1}+m, B_{2}+m, \ldots, B_{\ell(B)}+m\right\}$, therefore $A \mid B \in \Pi_{m+r}$ and this operation is noncommutative in the sense that, in general, $A|B \neq B| A$.

When writing examples of set partitions, whenever the context allows it, we will use a more compact notation. For example, $\{\{1,3,5\},\{2\},\{4\}\}$ will be represented by $\{135.2 .4\}$. Although there is no order on the parts of a set partition, we will impose an implied order such that the parts are arranged by increasing value of the smallest element in the subset. This implied order will allow us to reference the $i^{\text {th }}$ parts of the set partition without ambiguity.

There is a natural lattice structure on the set partitions of a given $n$. We define for $A, B \in \Pi_{n}$ that $A \leq B$ if for each $A_{i} \in A$ there is a $B_{j} \in B$ such that $A_{i} \subseteq B_{j}$ (otherwise stated, that $A$ is finer than $B)$. The set of set partitions of $[n]$ with this order forms a 
poset with rank function given by $n$ minus the length of the set partition. This poset has a unique minimal element $\mathbf{0}_{n}=\{1.2 \ldots . n\}$ and a unique maximal element $\mathbf{1}_{n}=\{12 \ldots n\}$. The largest element smaller than both $A$ and $B$ is denoted

$$
A \wedge B=\left\{A_{i} \cap B_{j}: 1 \leq i \leq \ell(A), 1 \leq j \leq \ell(B)\right\}
$$

while the smallest element larger than $A$ and $B$ is denoted $A \vee B$. The lattice $\left(\Pi_{n}, \wedge, \vee\right)$ is called the partition lattice.

Example 1.1 Let $A=\{138.24 .5 .67\}$ and $B=\{1.238 .4567\}$. $A$ and $B$ are not comparable in the inclusion order on set partitions. We calculate that $A \wedge B=\{1$ 2.38.4.5.67\} and $A \vee B=\{12345678\}$.

When a collection of disjoint sets of positive integers is not a set partition because the union of the parts is not $[n]$ for some $n$, we may lower the values in the sets so that they keep their relative values so that the resulting collection is a set partition (of an $m<n$ ). This operation is referred to as the 'standardization' of a set of disjoint sets $A$ and the resulting set partition is denoted $s t(A)$.

Now for $A \in \Pi_{m}$ and $S \subseteq\{1,2, \ldots, \ell(A)\}$ with $S=\left\{s_{1}, s_{2}, \ldots, s_{k}\right\}$, we define $A_{S}=$ $\operatorname{st}\left(\left\{A_{s_{1}}, A_{s_{2}}, \ldots, A_{s_{k}}\right\}\right)$ which is a set partition of $\left|A_{s_{1}}\right|+\left|A_{s_{2}}\right|+\ldots+\left|A_{s_{k}}\right|$. By convention $A_{\{\}}$is the empty set partition.

Example 1.2 If $A=\{1368.2 .4 .579\}$, then $A_{\{1,4\}}=\{1246.357\}$.

For $n \geq 0$, consider a set $X_{n}$ of non-commuting variables $x_{1}, x_{2}, \ldots, x_{n}$ and the polynomial algebra $\mathcal{R}_{X_{n}}=\mathbb{k}\left\langle x_{1}, x_{2}, \ldots, x_{n}\right\rangle$ in these non-commuting variables. There is a natural $S_{n}$ action on the basis elements defined by $\sigma\left(x_{i_{1}} x_{i_{2}} \cdots x_{i_{k}}\right)=x_{\sigma\left(i_{1}\right)} x_{\sigma\left(i_{2}\right)} \cdots x_{\sigma\left(i_{k}\right)}$. Let $x_{i_{1}} x_{i_{2}} \cdots x_{i_{m}}$ be a monomial in the space $\mathcal{R}_{X_{n}}$. We say that the type of this monomial is a set partition $A \in \Pi_{m}$ with the property that $i_{a}=i_{b}$ if and only if $a$ and $b$ are in the same block of the set partition. This set partition is denoted as $\nabla\left(i_{1}, i_{2}, \ldots, i_{m}\right)=A$. Notice that the length of $\nabla\left(i_{1}, i_{2}, \ldots, i_{m}\right)$ is equal to the number of different values which appear in $\left(i_{1}, i_{2}, \ldots, i_{m}\right)$.

The vector space NCSym ${ }^{(n)}$ is defined as the linear span of the elements

$$
\mathbf{m}_{A}\left[X_{n}\right]=\sum_{\nabla\left(i_{1}, i_{2}, \ldots, i_{m}\right)=A} x_{i_{1}} x_{i_{2}} \cdots x_{i_{m}}
$$

for $A \in \Pi_{m}$, where the sum is over all sequences with $1 \leq i_{j} \leq n$. For the empty set partition, we define by convention $\mathbf{m}_{\{\}}\left[X_{n}\right]=1$. If $\ell(A)>n$ we must have that $\mathbf{m}_{A}\left[X_{n}\right]=$ 0 . Since for any permutation $\sigma \in S_{n}, \nabla\left(i_{1}, i_{2}, \ldots, i_{m}\right)=\nabla\left(\sigma\left(i_{1}\right), \sigma\left(i_{2}\right), \ldots, \sigma\left(i_{m}\right)\right)$, we have that $\sigma \mathbf{m}_{A}\left[X_{n}\right]=\mathbf{m}_{A}\left[X_{n}\right]$. In fact, $\mathbf{m}_{A}\left[X_{n}\right]$ is the sum of all elements in the orbit of a monomial of type $A$ under the action of $S_{n}$. Therefore NCSym ${ }^{(n)}$ is the space of $S_{n^{-}}$ invariants in the noncommutative polynomial algebra $\mathcal{R}_{X_{n}}$. For instance, $\mathbf{m}_{\{13.2\}}\left[X_{4}\right]=$ $x_{1} x_{2} x_{1}+x_{1} x_{3} x_{1}+x_{1} x_{4} x_{1}+x_{2} x_{1} x_{2}+x_{2} x_{3} x_{2}+x_{2} x_{4} x_{2}+x_{3} x_{1} x_{3}+x_{3} x_{2} x_{3}+x_{3} x_{4} x_{3}+x_{4} x_{1} x_{4}+$ $x_{4} x_{2} x_{4}+x_{4} x_{3} x_{4}$. 
As in the classical case, where the number of variables is usually irrelevant as long as it is big enough, we want to consider that we have an infinite number of non-commuting variables. Since $\operatorname{NCSym}^{(n)}$ inherits from $\mathbb{k}\left\langle x_{1}, x_{2}, \ldots, x_{n}\right\rangle$ a graded algebra structure, we consider, for any $m \geq n$, the homomorphism of graded algebras $\mathbb{k}\left\langle x_{1}, \ldots, x_{m}\right\rangle \rightarrow \mathbb{k}\left\langle x_{1}, \ldots, x_{n}\right\rangle$ that sends variables $x_{n+1}, \ldots, x_{m}$ to zero and the remaining ones to themselves. This map restricts to a surjective homomorphism $\rho_{m, n}: \mathrm{NCSym}^{(m)} \rightarrow \mathrm{NCSym}^{(n)}$, that sends $\mathbf{m}_{A}\left[X_{m}\right]$ to $\mathbf{m}_{A}\left[X_{n}\right]$. The family $\left\{\operatorname{NCSym}^{(n)}: n \geq 1\right\}$ together with the homomorphisms $\rho_{m, n}$ forms an inverse system in the category of graded algebras. Let NCSym be its inverse limit in this category. We call NCSym the algebra of symmetric functions in an infinite number of non-commuting variables.

For each set partition $A$ there exits an unique element $\mathbf{m}_{A}$ whose projection to each NCSym ${ }^{(n)}$ is $\mathbf{m}_{A}\left[X_{n}\right]$. These elements are called monomial symmetric functions in an infinite number of non-commuting variables.

If we decompose NCSym as the sum of its graded pieces,

$$
\mathrm{NCSym}=\bigoplus_{d \geq 0} \mathrm{NCSym}_{d}
$$

then the monomial symmetric functions $\mathbf{m}_{A}$, with $A \vdash[d]$, is a linear basis of NCSym .

Here we forget any reference to the variables $x_{1}, x_{2}, \ldots$ and think of elements in NCSym as noncommutative symmetric functions. The degree of a basis element $\mathbf{m}_{A}$ is given by $|A|=d$ and the product map $\mu: \mathrm{NCSym}_{d} \otimes \mathrm{NCSym}_{m} \longrightarrow \mathrm{NCSym}_{d+m}$ is defined on the basis elements $\mathbf{m}_{A} \otimes \mathbf{m}_{B}$ by

$$
\mu\left(\mathbf{m}_{A} \otimes \mathbf{m}_{B}\right):=\sum_{\substack{C \in \Pi_{d+m} \\ C \wedge \mathbf{1}_{d}\left|\mathbf{1}_{m}=A\right| B}} \mathbf{m}_{C} .
$$

This is a lift of the multiplication in $\operatorname{NCSym}^{(n)}$.

The graded algebra NCSym is in fact a Hopf algebra with the following comultiplication $\Delta: \mathrm{NCSym}_{d} \longrightarrow \bigoplus_{k=0}^{d} \mathrm{NCSym}_{k} \otimes \mathrm{NCSym}_{d-k}$ where

$$
\Delta\left(\mathbf{m}_{A}\right)=\sum_{S \subseteq[\ell(A)]} \mathbf{m}_{A_{S}} \otimes \mathbf{m}_{A_{S^{c}}}
$$

and $S^{c}=[\ell(A)]-S$. The counit is given by $\epsilon:$ NCSym $\rightarrow \mathbb{Q}$ where $\epsilon\left(\mathbf{m}_{\{\}}\right)=1$ and $\epsilon\left(\mathbf{m}_{A}\right)=0$ for all $A \in \Pi_{n}$ for $n>0$. More details on this Hopf algebra structure are found in $[7,8]$.

The algebra NCSym was originally considered by Wolf [21] in extending the fundamental theorem of symmetric functions to this algebra and later by Bergman and Cohn [9]. More recently Rosas and Sagan [17] considered this space to define natural bases which are analogous to bases of the (commutative) symmetric functions. More progress in understanding this space was made in $[7,8]$ where it was considered as a Hopf algebra. In the Hopf algebra Sym of (commutative) symmetric functions, the comultiplication corresponds to the plethysm $f[X] \mapsto f[X+Y]$. It was established in [7] that the comultiplication in NCSym corresponds to a noncommutative plethysm $F[X] \mapsto F[X+Y]$, where 
$X+Y$ is the alphabet (totally ordered set of non-commuting variables) corresponding to the disjoint union of $X$ and $Y$, together with the total order obtained from $X$ and $Y$ placing all $Y$ after all $X$. (That is, $x<y$ for all $x$ in $X$ and all $y$ in $Y$.)

The Hopf algebra Sym has more structure. There is a second comultiplication corresponding to the plethysm $f[X] \mapsto f[X Y]$ (see $[16,22]$ ). This second operation is often referred to as the internal comultiplication or Kronecker comultiplication. We end this section describing for NCSym the analog of this internal comultiplication. This description is also considered in [2].

For the Hopf algebra NCSym we define a second (internal) comultiplication

$$
\Delta^{\odot}: \mathrm{NCSym}_{d} \longrightarrow \mathrm{NCSym}_{d} \otimes \mathrm{NCSym}_{d}
$$

by

$$
\Delta^{\odot}\left(\mathbf{m}_{A}\right)=\sum_{B \wedge C=A} \mathbf{m}_{B} \otimes \mathbf{m}_{C} .
$$

This operation corresponds to a noncommutative plethysm $F[X] \mapsto F[X Y]$. More precisely, assume that we have two countable alphabet $X=x_{1}, x_{2}, \ldots$ and $Y=y_{1}, y_{2}, \ldots$ Then, $X Y=x_{1} y_{1}, x_{1} y_{2}, \ldots, x_{i} y_{j}, \ldots$, totally ordered using the lexicographic order. That is, $x y<z w$ if and only if $(x<z)$ or $(x=z$ and $y<w)$ for all $x, z$ in $X$ and all $y, w$ in $Y$. We conclude that the transformation $F[X] \mapsto F[X Y]$ sends $F\left(x_{1}, x_{2}, \ldots\right)$ to $F\left(x_{1} y_{1}, x_{1} y_{2}, \ldots, x_{2} y_{1}, x_{2} y_{2}, \ldots\right)$.

If we let the $x_{i}$ 's commute with the $y_{j}$ 's then we have that $F[X Y]$ can be expanded in the form $F[X Y]=\sum F_{1, i}[X] F_{2, i}[Y]$. We can then define the operation

$$
\Delta^{\odot}(F)=\sum F_{1, i} \otimes F_{2, i} .
$$

Equation (3) gives the result of this when $F=\mathbf{m}_{A}$. Clearly this operation is a morphism for the multiplication, thus NCSym with $\Delta^{\odot}$ and the multiplication operation of equation (1) forms a bialgebra. But it is not a Hopf algebra as it does not have an antipode. We are now in position to state our first main theorem.

Remark: In order to define the sum and product of two alphabets, $X+Y$ and $X Y$, on the inverse limit of $k\left\langle x_{1}, \ldots, x_{n}\right\rangle$, it is necessary to introduce a total order on each of them. On the other hand, when we restrict ourselves to elements of Sym, the result is independent of the particular choice of total order we made.

Theorem 1.3 There is a basis $\left\{\mathbf{x}_{A}: A \in \Pi_{n}, n \geq 0\right\}$ of NCSym such that

(i) $\mathbf{x}_{A} \mathbf{x}_{B}=\mathbf{x}_{A \mid B}$

(ii) $\Delta^{\odot}\left(\mathbf{x}_{C}\right)=\sum_{A \vee B=C} \mathbf{x}_{A} \otimes \mathbf{x}_{B}$.

The proof of this theorem is technical and we differ it to Section 4. We are convinced that the basis $\left\{\mathbf{x}_{A}: A \in \Pi_{n}, n \geq 0\right\}$ is central in the study of NCSym and should have many fascinating properties. We plan to study this basis further in future work. For now, we prefer to develop the representation theory that will motivate our result. 


\section{Grothendieck bialgebra of the Semi-tower $(\Pi, \wedge)=$ $\bigoplus_{n \geq 0}\left(\mathbb{k} \Pi_{n}, \wedge\right)$.}

In this section we consider the partition lattice algebras. For a fixed $n$ consider the vector space $\left(\mathbb{k} \Pi_{n}, \wedge\right)$ formally spanned by the set partitions of $n$. The multiplication is given by the operation $\wedge$ on set partitions and with the unit $\mathbf{1}_{n}=\{1,2, \ldots, n\}$. We remark that for all $d$, we have that $\mathbb{k} \Pi_{d}$ is isomorphic as a vector space to $\mathrm{NCSym}_{d}$ via the pairing $A \leftrightarrow \mathbf{m}_{A}$. Moreover, it is straightforward to check using equation (3) that $\Delta^{\odot}$ is dual to $\wedge$ as operators.

It is well known that $\left(\mathbb{k}_{n}, \wedge\right)$ is a commutative semisimple algebra (see $[19$, Theorem 3.9.2]). To see this, one considers the algebra $\mathbb{k}^{\Pi_{n}}=\left\{f: \Pi_{n} \rightarrow \mathbb{k}\right\}$ which is clearly commutative and semisimple. We then define the map

$$
\begin{aligned}
\delta_{\geq}:\left(\mathbb{k}_{n}, \wedge\right) & \rightarrow \mathbb{k}^{\Pi_{n}} \\
A & \mapsto \delta_{A \geq},
\end{aligned}
$$

where $\delta_{A \geq}(B)=1$ if $A \geq B$ and 0 otherwise. Next check that $\delta_{A \wedge B \geq}=\delta_{A \geq} \delta_{B \geq}$ which shows that $\delta_{\geq}$is an isomorphism of algebras.

The primitive orthogonal idempotents of $\mathbb{k}^{\Pi_{n}}$ are given by the functions $\delta_{A=}$ defined by $\delta_{A=}(B)=1$ if $A=B$ and 0 otherwise. We have that $\delta_{A \geq}=\sum_{B \leq A} \delta_{B=}$. This implies, using Möbius inversion, that the primitive orthogonal idempotents of $\left(\mathbb{k} \Pi_{n}, \wedge\right)$ are given by

$$
e_{A}=\sum_{B \leq A} \mu(B, A) B
$$

where $\mu$ is the Möbius function of the partially ordered set $\Pi_{n}$. Since $\left(\mathbb{k} \Pi_{n}, \wedge\right)$ is commutative and semisimple, we have that the simple $\left(\mathbb{k}_{n}, \wedge\right)$-modules of this algebra are the one dimensional spaces $V_{A}=\mathbb{k} \Pi_{n} \wedge e_{A}$. Here the action is given by the left multiplication

$$
C \wedge e_{A}= \begin{cases}e_{A} & \text { if } C \geq A, \\ 0 & \text { otherwise }\end{cases}
$$

This follows from the corresponding identity in $\mathbb{k}^{\Pi_{n}}$ considering $\delta_{\geq C} \delta_{=A}$.

We now let $G_{0}\left(\mathbb{k}_{n}, \wedge\right)$ denote the Grothendieck group of the category of finite dimensional $\left(\mathbb{k} \Pi_{n}, \wedge\right)$-modules. This is the vector space spanned by the equivalence classes of simple $\left(\mathbb{k} \prod_{n}, \wedge\right)$-modules under isomorphisms.

We also consider $K_{0}\left(\mathbb{k}_{n}, \wedge\right)$ the Grothendieck group of the category of projective $\left(\mathbb{k} \Pi_{n}, \wedge\right)$-modules. Since $\left(\mathbb{k} \Pi_{n}, \wedge\right)$ is semisimple, the space $G_{0}\left(\mathbb{k} \Pi_{n}, \wedge\right)$ and $K_{0}\left(\mathbb{k} \Pi_{n}, \wedge\right)$ are equal as vector spaces as they are both linearly spanned by the elements $V_{A}$ for $A \in \Pi_{n}$. We then set $K_{0}(\boldsymbol{\Pi}, \wedge)=\bigoplus_{n \geq 0} K_{0}\left(\mathbb{k} \Pi_{n}, \wedge\right)$.

Given two finite $\left(\mathbb{k}_{n}, \wedge\right)$ modules $V$ and $W$, we can form the $\left(\mathbb{k} \Pi_{n}, \wedge\right)$-module $V \otimes W$ with the diagonal action (it is an action since a semigroup algebra is a bialgebra for the coproduct $A \rightarrow A \otimes A)$. We denote this $\left(\mathbb{k} \Pi_{n}, \wedge\right)$-module by $V \odot W$ (to avoid confusion with the tensor product of a $\left(\mathbb{k} \Pi_{n}, \wedge\right)$-module and a $\left(\mathbb{k} \Pi_{m}, \wedge\right)$-module). 
Lemma 2.1 Given two simple $\left(\mathbb{k} \Pi_{n}, \wedge\right)$-module $V_{A}$ and $V_{B}$,

$$
V_{A} \odot V_{B}=V_{A \vee B}
$$

proof: Let $C \in \Pi_{n}$ act on $e_{A} \otimes e_{B}$. From equation (5) we get $C \wedge\left(e_{A} \otimes e_{B}\right)=$ $\left(C \wedge e_{A}\right) \otimes\left(C \wedge e_{B}\right)=e_{A} \otimes e_{B}$ if and only if $C \geq A$ and $C \geq B$, that is $C \geq A \vee B$. If not, we get $C \wedge\left(e_{A} \otimes e_{B}\right)=0$. We conclude that the map $e_{A} \otimes e_{B} \mapsto e_{A \vee B}$ is the desired isomorphism in equation (6).

We would like to define on $G_{0}(\Pi, \wedge)=\bigoplus_{n \geq 0} G_{0}\left(\mathbb{k} \Pi_{n}, \wedge\right)$ a graded multiplication and a graded comultiplication corresponding to induction and restriction. For this we need a few more tools.

Lemma 2.2 The linear map $\rho_{n, m}:\left(\mathbb{k}_{n}, \wedge\right) \otimes\left(\mathbb{k}_{m}, \wedge\right) \rightarrow\left(\mathbb{k}_{n+m}, \wedge\right)$ defined by

$$
\rho_{n, m}(A \otimes B)=A \mid B
$$

is injective and multiplicative. Moreover, $\rho_{k+n, m} \circ\left(\rho_{k, n} \otimes I d\right)=\rho_{k, n+m} \circ\left(I d \otimes \rho_{n, m}\right)$ for all $k, n$ and $m$.

proof: Let $A=\left\{A_{1}, \ldots, A_{r}\right\}, B=\left\{B_{1}, \ldots, B_{s}\right\}$ be set partitions in $\Pi_{n}$, and $C=$ $\left\{C_{1}, \ldots, C_{t}\right\}$ and $D=\left\{D_{1}, \ldots, D_{u}\right\}$ be set partitions in $\Pi_{m}$. We remark that for all $i, j$, we have $A_{i} \cap\left(D_{j}+n\right)=\emptyset$ and $\left(C_{i}+n\right) \cap B_{j}=\emptyset$. Since $\left(C_{i}+n\right) \cap\left(D_{j}+n\right)=\left(C_{i} \cap D_{j}\right)+n$, we have

$$
\begin{aligned}
(A \mid C) \wedge(B \mid D) & =\left\{A_{i} \cap B_{j}\right\}_{\substack{1 \leq i \leq r \\
1 \leq j \leq s}} \cup\left\{\left(C_{i}+n\right) \cap\left(D_{j}+n\right)\right\}_{\substack{1 \leq i \leq t \\
1 \leq j \leq u}} \\
& =(A \wedge B) \mid(C \wedge D),
\end{aligned}
$$

and this shows that $\rho_{n, m}$ is multiplicative. The injectivity of this map is clear from the fact that $\rho_{n, m}$ maps distinct basis elements into distinct basis elements. The last identity of the lemma follows from the associativity of the operation "|"

We define a semi-tower $\left(\bigoplus_{n>0} A_{n},\left\{\phi_{n, m}\right\}\right)$ to be a direct sum of algebras along with a family of injective non-unital homomorphisms of algebras $\phi_{n, m}: A_{n} \otimes A_{m} \rightarrow A_{n+m}$. A tower in the sense defined in the recent literature $[5,12,15]$ is a semi-tower with the additional constraint that $\phi_{n, m}\left(\mathbf{1}_{n}, \mathbf{1}_{m}\right)=\mathbf{1}_{n+m}$ (i.e. that $\phi_{n, m}$ is a unital embedding of algebras).

Define the pair $(\Pi, \wedge)=\left(\bigoplus_{n \geq 0}\left(\mathbb{k} \Pi_{n}, \wedge\right),\left\{\rho_{n, m}\right\}\right)$ which is a semi-tower of the algebras $\left(\mathbb{k}_{n}, \wedge\right)$. We remark that $(\Pi, \wedge)$ is a graded algebra with the multiplication $\rho_{n, m}(A, B)=A \mid B$ which is associative (but non-commutative) and has a unit given by the emptyset partition $\emptyset \in \Pi_{0}$. Moreover, each of the homogeneous components $\left(\mathbb{k}_{n}, \wedge\right)$ of $\boldsymbol{\Pi}$ are themselves algebras with the multiplication $\wedge$, and Lemma 2.2 gives the relationship between the two operations.

At this point we need to stress that $\rho_{n, m}$ is not a unital embedding of algebras and hence $(\boldsymbol{\Pi}, \wedge)$ is not a tower of algebras. The algebra $\left(\mathbb{k} \Pi_{n}, \wedge\right)$ has a unit given by $\mathbf{1}_{n}=\{12 \ldots n\}$, 
but $\rho_{n, m}\left(\mathbf{1}_{n} \otimes \mathbf{1}_{m}\right) \neq \mathbf{1}_{n+m}$. The tower of algebras considered in the recent literature $[5,12,15]$ all have the property that the corresponding $\rho_{n, m}$ are (unital) embeddings of algebras. This is the reason we call our construction a semi-tower rather than a tower.

The motivation for defining a tower of algebras is to allow one to induce and restrict modules of these algebras and ultimately to define on its Grothendieck ring a Hopf algebra structure. Here the fact that we have only a semi-tower causes some problems in defining restriction of modules. Yet we can still define a weaker version of restriction in our situation. Let $A$ and $B$ be two finite dimensional algebras and let $\rho: A \rightarrow B$ be a multiplicative injective linear map. Given a finite $B$-module $M$, we define

$$
\operatorname{Res}_{\rho} M=\left\{m \in M: \rho\left(\mathbf{1}_{A}\right) m=m\right\} \subseteq M \text {. }
$$

In the case where $\rho$ is an embedding of algebras this definition agrees with the traditional one. More on this general theory will be found in [14] but here we focus our attention on $(\Pi, \wedge)$.

Lemma 2.3 For $k \leq n$ and a simple $\left(\mathbb{k} \Pi_{n}, \wedge\right)$-module $V_{A} \in G_{0}\left(\mathbb{k} \Pi_{n}, \wedge\right)$,

$$
\operatorname{Res}_{\rho_{k, n-k}} V_{A}= \begin{cases}V_{A} & \text { if } A=B \mid C \text { for } B \in \Pi_{k} \text { and } C \in \Pi_{n-k} \\ 0 & \text { otherwise. }\end{cases}
$$

proof: We have that $\rho_{n, m}\left(\mathbf{1}_{k} \otimes \mathbf{1}_{n-k}\right) \wedge e_{A}=\left(\mathbf{1}_{k} \mid \mathbf{1}_{n-k}\right) \wedge e_{A}=e_{A}$ if $\mathbf{1}_{k} \mid \mathbf{1}_{n-k} \geq A$, and 0 otherwise. The condition $\mathbf{1}_{k} \mid \mathbf{1}_{n-k} \geq A$ is equivalent to $A=B \mid C$ where $\left.A\right|_{1, \ldots, k}=B$ and $\left.A\right|_{k+1, \ldots, n+k}=C$.

We can now define a graded comultiplication on $G_{0}(\boldsymbol{\Pi}, \wedge)$ using our definition of restriction. For $V \in G_{0}\left(\mathbb{k} \Pi_{n}, \wedge\right)$ let

$$
\Delta(V)=\sum_{k=0}^{n} \operatorname{Res}_{\rho_{k, n-k}} V .
$$

It follows from Lemmas 2.2 that this operation is coassociative. For a simple module $V_{A} \in G_{0}\left(\mathbb{k} \Pi_{n}, \wedge\right)$, Lemma 2.3 gives us

$$
\Delta\left(V_{A}\right)=\sum_{A=B \mid C} V_{B} \otimes V_{C}
$$

Now we extend $\odot$ to $G_{0}(\Pi, \wedge)$ by setting $V_{A} \odot V_{B}=0$ if $V_{A}$ and $V_{B}$ are not of the same degree.

Proposition $2.4\left(G_{0}(\Pi, \wedge), \odot, \Delta\right)$ is a bialgebra.

proof: Let $A, B \in \Pi_{n}$. By equation (6), it is sufficient to prove that $\Delta\left(V_{A \vee B}\right)=$ $\Delta\left(V_{A}\right) \odot \Delta\left(V_{B}\right)$. Using equation $(2.3)$ we can easily reduce the problem to the following assertion: there are $C \in \Pi_{k}, D \in \Pi_{n-k}$ such that $A \vee B=C \mid D$ if and only if there are 
$E, E^{\prime} \in \Pi_{k}, F, F^{\prime} \in \Pi_{n-k}$ such that $A=E \mid F$ and $B=E^{\prime} \mid F^{\prime}$. This follows then from definitions.

It is thus natural to give a notion to induced modules dual to restriction in Lemma 2.3.

Lemma 2.5 For two simple modules $V_{A}=\mathbb{k} \Pi_{n} \wedge e_{A} \in G_{0}\left(\mathbb{k}_{n}, \wedge\right)$ and $V_{B}=\mathbb{k}_{m} \wedge e_{B} \in$ $G_{0}\left(\mathbb{k} \Pi_{m}, \wedge\right)$ we define

$$
\operatorname{Ind}_{n, m} V_{A} \otimes V_{B}=\mathbb{k}_{n+m} \otimes_{\mathbb{k} \Pi_{n} \otimes \mathbb{k} \Pi_{m}}\left(\mathbb{k} \Pi_{n} \wedge e_{A} \otimes \mathbb{k} \Pi_{m} \wedge e_{B}\right),
$$

where $\mathbb{k}_{n} \otimes \mathbb{k}_{m}$ is embedded into $\mathbb{k} \Pi_{n+m}$ via $\rho_{n, m}$.

There is a natural isomorphism such that

$$
\operatorname{Ind}_{n, m} V_{A} \otimes V_{B} \cong \mathbb{k} \Pi_{n+m} \wedge \rho_{n, m}\left(e_{A} \otimes e_{B}\right) .
$$

We have

$$
\operatorname{Ind}_{n, m} V_{A} \otimes V_{B}=V_{A \mid B} .
$$

proof: Consider the following isomorphism which allows us to naturally realize

$$
\operatorname{Ind}_{n, m} V_{A} \otimes V_{B}
$$

as an element of $G_{0}\left(\mathbb{k} \Pi_{n+m}, \wedge\right)$.

$$
\begin{aligned}
\operatorname{Ind}_{n, m} V_{A} \otimes V_{B} & =\mathbb{k} \Pi_{n+m} \otimes_{\mathbb{k} \Pi_{n} \otimes \mathbb{k} \Pi_{m}}\left(\mathbb{k} \Pi_{n} \wedge e_{A} \otimes \mathbb{k}_{m} \wedge e_{B}\right) \\
& =\mathbb{k} \Pi_{n+m} \otimes_{\mathbb{k} \Pi_{n} \otimes \mathbb{k} \Pi_{m}}\left(e_{A} \otimes e_{B}\right) \\
& =\mathbb{k} \Pi_{n+m} \wedge \rho_{n, m}\left(e_{A} \otimes e_{B}\right) \otimes_{\mathbb{k} \Pi_{n} \otimes \mathbb{k} \Pi_{m}}\left(\mathbf{1}_{n} \otimes \mathbf{1}_{n}\right) \\
& \cong \mathbb{k} \Pi_{n+m} \wedge \rho_{n, m}\left(e_{A} \otimes e_{B}\right) .
\end{aligned}
$$

By linearity

$$
\rho_{n, m}\left(e_{A} \otimes e_{B}\right)=e_{A}\left|e_{B}=\sum_{C \leq A} \sum_{D \leq B} \mu(C, A) \mu(D, B) C\right| D .
$$

We now remark that $\{E: E \leq A \mid B\}=\{C|D: C| D \leq A \mid B\}=\{C \mid D: C \leq A, D \leq B\}$. This is isomorphic to the cartesian product $\{C: C \leq A\} \times\{D: D \leq B\}$. Since Möbius functions are multiplicative with respect to cartesian product we have

$$
\rho_{n, m}\left(e_{A} \otimes e_{B}\right)=\sum_{E \leq A \mid B} \mu(E, A \mid B) E=e_{A \mid B}
$$

It is clear now that $\operatorname{Ind}_{n, m}$ defines on $G_{0}(\Pi, \wedge)$ a graded multiplication $V_{A} \otimes V_{B} \mapsto V_{A \mid B}$ that is dual to the graded comultiplication of $\Delta$ defined on $G_{0}(\boldsymbol{\Pi}, \wedge)$. We also define an internal comultiplication on $G_{0}(\Pi, \wedge)$ dual to equation $(6)$ such that $\Delta^{\odot}: G_{0}\left(\mathbb{k}_{n}, \wedge\right) \rightarrow$ $G_{0}\left(\mathbb{k} \Pi_{n}, \wedge\right) \otimes G_{0}\left(\mathbb{k} \Pi_{n}, \wedge\right)$. For $C \in \Pi_{n}$ let

$$
\Delta^{\odot}\left(V_{C}\right)=\sum_{A \vee B=C} V_{A} \otimes V_{B} .
$$

The space $G_{0}(\boldsymbol{\Pi}, \wedge)$ with its graded multiplication given by induction and comultiplication $\Delta^{\odot}$ is a bialgebra, by duality and Proposition 2.4. The main theorem of this section is a direct corollary to Theorem 1.3. 
Theorem 2.6 The map $F: G_{0}(\Pi, \wedge) \rightarrow$ NCSym defined by

$$
F\left(V_{A}\right)=\mathbf{x}_{A}
$$

is an isomorphism of bialgebras.

proof: $G_{0}(\Pi, \wedge)$ is endowed with a product given by $(9)$ and an inner coproduct given by (10). Since NCSym is known to be a bialgebra satisfying the relations given in Theorem 1.3 , the map $F$ is an isomorphism.

The map $F$ is called the Frobenius map for our semi-tower. Along with Theorem 1.3, it shows that the basis $\mathbf{x}_{A}$ of NCSym are the only functions that correspond to the classes of simple modules in $G_{0}(\boldsymbol{\Pi}, \wedge)$. This defines $\mathbf{x}_{A}$ uniquely (up to automorphism) and for this reason we think of them as the Schur functions for the semi-tower $(\boldsymbol{\Pi}, \wedge)$ of the symmetric functions in non-commutative variables.

Remark 2.7 In [10], Brown shows that $\left(\mathbb{k} \Pi_{n}, \wedge\right)$ is the semisimple quotient of the SolomonTits algebra $S T_{n}$ (see [20]). It is easy to lift our semi-tower structure from $\boldsymbol{\Pi}$ to $\mathbf{S T}=$ $\bigoplus S T_{n}$ via Brown's support map. Then $G_{0}(\mathbf{S T}, \wedge)$ and $G_{0}(\boldsymbol{\Pi}, \wedge)$ are isomorphic as bialgebras.

In [14], the conditions under which a tower of algebras $\mathbf{A}=\left(\bigoplus_{n>0} A_{n}, \rho_{n, m}\right)$ defines a Hopf algebra structure on the Grothendieck rings $G_{0}(\mathbf{A})$ and $K_{0}(\mathbf{A})$ are considered. Under certain conditions one would expect that the Grothendieck ring $G_{0}(\mathbf{A})$ of finite modules forms a Hopf algebra with the operations of induction and restriction which is isomorphic to the graded dual of the Grothendieck ring $K_{0}(\mathbf{A})$ of projective modules.

For the tower of algebras we are considering here, it is not the case that $G_{0}(\Pi, \wedge)$ forms a Hopf algebra because the operations of induction and restriction are not even compatible as a bialgebra structure. We have shown that $G_{0}(\Pi, \wedge)$ and $K_{0}(\Pi, \wedge)$ are endowed naturally with a product given by the notion of induction in equation (9) and coproduct given by the notion of restriction given in equation (7). It is easily checked that these operations do not form a Hopf algebra structure.

We have found however that here we have $G_{0}(\Pi, \wedge)$ endowed with the operations of induction and restriction is isomorphic by the graded dual to $K_{0}(\Pi, \wedge)$ also endowed with the same induction and restriction operations. This is because the operation of restriction on $G_{0}(\Pi, \wedge)$ is dual to the operation of induction on $K_{0}(\Pi, \wedge)$ and induction on $G_{0}(\Pi, \wedge)$ is dual as graded operations to restriction on $K_{0}(\boldsymbol{\Pi}, \wedge)$. This remark can be observed through the duality in equations (9) and (7).

\section{Grothendieck bialgebras of the Tower $(\Pi, \vee)=$ $\bigoplus_{n \geq 0}\left(\mathbb{k} \Pi_{n}, \vee\right)$.}

In this section we consider a second algebra related to the partition lattice and show that there is an additional connection with the algebra NCSym. Define $\left(\mathbb{k}_{k} \Pi_{n}, \vee\right)$ to be 
the commutative algebra linearly spanned by the elements of $\Pi_{n}$ and endowed with the product $\vee$. This algebra has as a unit the minimal element $\mathbf{0}_{n}=\{1.2 . \cdots . n\}$ of the poset $\Pi_{n}$ since $\mathbf{0}_{n} \vee A=A$ for all $A \in \Pi_{n}$.

As we constructed the primitive orthogonal idempotents for $\left(\mathbb{k} \Pi_{n}, \wedge\right)$, we proceed by defining in a similar manner

$$
\begin{aligned}
\delta_{\leq}:\left(\mathbb{k} \Pi_{n}, \vee\right) & \rightarrow \mathbb{k}^{\Pi_{n}} \\
A & \mapsto \delta_{A \leq} .
\end{aligned}
$$

It is straightforward to check that $\delta_{A \leq} \delta_{B \leq}=\delta_{(A \vee B)} \leq$ and hence $\delta_{\leq}$is an isomorphism of algebras. This map can be used to recover the primitive orthogonal idempotents of $\left(\mathbb{k} \Pi_{n}, \vee\right)$ since if $\delta_{A \leq}=\sum_{B \geq A} \delta_{B=}$, then $\delta_{A=}=\sum_{B \geq A} \mu(A, B) \delta_{B \leq}$. This can be summarized in the following proposition.

Proposition 3.1 The primitive orthogonal idempotents of the algebra $\left(\mathbb{k} \Pi_{n}, \vee\right)$ are

$$
f_{A}=\sum_{B \geq A} \mu(A, B) B
$$

with the property that

$$
C \vee f_{A}= \begin{cases}f_{A} & \text { if } C \leq A \\ 0 & \text { otherwise }\end{cases}
$$

It is also not difficult to check that the map $\rho_{n, m}(A, B)=A \mid B$ is also multiplicative with respect to the $V$ product in analogy with Lemma 2.2. Therefore we define the tower of algebras $(\Pi, \vee)=\left(\bigoplus_{n>0}\left(\mathbb{k} \Pi_{n}, \vee\right),\left\{\rho_{n, m}\right\}\right)$. This time we find that $\rho_{n, m}$ is indeed an embedding of algebras and $(\boldsymbol{\Pi}, \vee)$ a tower of algebras (see remarks related to $(\boldsymbol{\Pi}, \wedge)$ ) since $\rho_{n, m}\left(\mathbf{0}_{n}, \mathbf{0}_{m}\right)=\mathbf{0}_{n+m}$.

We now define $G_{0}\left(\mathbb{k} \Pi_{n}, \vee\right)$ to be the ring of the category of finite dimensional $\left(\mathbb{k} \Pi_{n}, \vee\right)$ modules endowed with the tensor of modules as the product. $G_{0}\left(\mathbb{k} \Pi_{n}, \vee\right)$ is linearly spanned by the equivalence classes of the simple modules under isomorphism. Also set $K_{0}\left(\mathbb{k} \Pi_{n}, \vee\right)$ to be the Grothendieck ring of the category of projective $\left(\mathbb{k} \Pi_{n}, \vee\right)$-modules. Since $\left(\mathbb{k} \Pi_{n}, \vee\right)$ is semi-simple we find that $G_{0}\left(\mathbb{k} \Pi_{n}, \vee\right) \cong K_{0}\left(\mathbb{k} \Pi_{n}, \vee\right)$ and both are spanned by the simple modules $W_{A}=\mathbb{k} \Pi_{n} \vee f_{A}$. Set $G_{0}(\Pi, \vee)=\bigoplus_{n \geq 0} G_{0}\left(\mathbb{k} \Pi_{n}, \vee\right)$ and $K_{0}(\Pi, \vee)=$ $\bigoplus_{n \geq 0} K_{0}\left(\mathbb{k} \Pi_{n}, \vee\right)$.

Given two simple modules $W_{A}, W_{B} \in G_{0}\left(\mathbb{k} \Pi_{n}, \vee\right)$ we consider the tensor product of modules $W_{A} \otimes W_{B}$ with the diagonal action of $\left(\mathbb{k} \Pi_{n}, \vee\right)$. Denote this module as $W_{A} \odot W_{B}$. We find that $C \vee\left(f_{A} \otimes f_{B}\right)=\left(C \vee f_{A}\right) \otimes\left(C \vee f_{B}\right)$ which is equal to $f_{A} \otimes f_{B}$ if $C \leq A$ and $C \leq B$ (i.e. $C \leq A \wedge B$ ) and it is equal to 0 otherwise. We conclude from this discussion the following lemma.

Lemma 3.2 For $W_{A}, W_{B} \in G_{0}\left(\mathbb{k} \Pi_{n}, \vee\right)$,

$$
W_{A} \odot W_{B}=W_{A \wedge B}
$$

is a simple module. 
We have the following formula for the restriction of $W_{A}$ to $\mathbb{k} \prod_{k} \otimes \mathbb{k} \prod_{n-k}$.

Lemma 3.3 For $k \leq n$ and a simple module $W_{A} \in G_{0}\left(\mathbb{k} \Pi_{n}, \vee\right)$,

$$
\operatorname{Res}_{\rho_{k, n-k}} W_{A}=W_{B} \otimes W_{C}
$$

where $A \wedge\left(\mathbf{1}_{k} \mid \mathbf{1}_{n-k}\right)=B \mid C$ for $B \in \Pi_{k}$ and $C \in \Pi_{n-k}$.

proof: First we check that $\rho_{n, m}\left(\mathbf{0}_{k} \otimes \mathbf{0}_{n-k}\right) f_{A}=\mathbf{0}_{n} \vee f_{A}=f_{A}$. Now for $B^{\prime} \in \Pi_{k}$ and $C^{\prime} \in \Pi_{n-k}$, we have that $\rho_{k, n-k}\left(B^{\prime}, C^{\prime}\right) \vee f_{A}=\left(B^{\prime} \mid C^{\prime}\right) \vee f_{A}=f_{A}$ if $\left(B^{\prime} \mid C^{\prime}\right) \leq A$ and 0 otherwise. If $A \wedge\left(\mathbf{1}_{k} \mid \mathbf{1}_{n-k}\right)=(B \mid C)$ then $\left(B^{\prime} \mid C^{\prime}\right) \vee f_{A}=f_{A}$ if and only if $B^{\prime} \leq B$ and $C^{\prime} \leq C$. Therefore $W_{A}$ is isomorphic to $W_{B} \otimes W_{C}$ as a $\mathbb{k} \prod_{k} \otimes \mathbb{k} \Pi_{n-k}$ module.

Define now a notion of induction for $K_{0}(\Pi, \vee)$ (as the dual of $G_{0}(\Pi, \vee)$ ). For $A \in \Pi_{n}$ and $B \in \Pi_{m}$, the induced $\left(\mathbb{k} \Pi_{n+m}, \vee\right)$ module is

$$
\operatorname{Ind}_{n, m} W_{A} \otimes W_{B}=\mathbb{k} \Pi_{n+m} \otimes_{\mathbb{k} \Pi_{n} \otimes \mathbb{k} \Pi_{m}}\left(W_{A} \otimes W_{B}\right)
$$

where we consider $\mathbb{k}_{n} \otimes \mathbb{k} \Pi_{m} \cong \rho_{n, m}\left(\mathbb{k} \Pi_{n} \otimes \mathbb{k} \Pi_{m}\right) \subseteq \mathbb{k} \Pi_{n+m}$.

Lemma 3.4 For $A \in \Pi_{n}$ and $B \in \Pi_{m}$ we have that

$$
\operatorname{Ind}_{n, m} W_{A} \otimes W_{B}=\sum_{C \wedge\left(\mathbf{1}_{n} \mid \mathbf{1}_{m}\right)=A \mid B} W_{C} .
$$

proof: By proceeding as in the proof of Lemma 2.5, we get

$$
\operatorname{Ind}_{n, m} W_{A} \otimes W_{B} \cong \mathbb{k} \Pi_{n+m} \wedge \rho_{n, m}\left(f_{A} \otimes f_{B}\right) .
$$

Therefore we just have to prove

$$
\rho_{n, m}\left(f_{A} \otimes f_{B}\right)=\sum_{C \wedge\left(\mathbf{1}_{n} \mid \mathbf{1}_{m}\right)=A \mid B} f_{C}
$$

Since Möbius functions are multiplicative with respect to cartesian product we have on the one hand

$$
\rho_{n, m}\left(f_{A} \otimes f_{B}\right)=\sum_{E|F \geq A| B} \mu(A|B, E| F) E \mid F .
$$

On the other hand

$$
\begin{aligned}
\sum_{C \wedge\left(\mathbf{1}_{n} \mid \mathbf{1}_{m}\right)=A \mid B} f_{C}= & \sum_{C \wedge\left(\mathbf{1}_{n} \mid \mathbf{1}_{m}\right)=A \mid B} \sum_{D \geq C} \mu(C, D) D \\
= & \sum_{E|F \geq A| B} \sum_{C \wedge\left(\mathbf{1}_{n} \mid \mathbf{1}_{m}\right)=A \mid B} \sum_{\substack{D \wedge\left(\mathbf{1}_{n} \mid \mathbf{1}_{m}\right)=E \mid F \\
D \geq C}} \mu(C, D) D \\
= & \sum_{E|F \geq A| B} \mu(A|B, E| F) E \mid F \\
& +\sum_{E|F \geq A| B} \sum_{\substack{D \wedge\left(\mathbf{1}_{n} \mid \mathbf{1}_{m}\right)=E|F \\
D \neq E| F}}\left(\sum_{\substack{C \wedge\left(\mathbf{1}_{n} \mid \mathbf{1}_{m}\right)=A \mid B \\
C \leq D}} \mu(C, D)\right) D .
\end{aligned}
$$


The result follows then from the following equality

$$
\sum_{\substack{C \wedge\left(\mathbf{1}_{n} \mid \mathbf{1}_{m}\right)=A \mid B \\ C \leq D}} \mu(C, D)=\sum_{A \mid B \leq C \leq D} \mu(C, D)=0 .
$$

Induction and restriction define a graded product and coproduct on the space of $G_{0}(\Pi, \vee)=\bigoplus_{n \geq 0} G_{0}\left(\mathbb{k} \Pi_{n}, \vee\right)$. Define on the elements $N \in G_{0}\left(\mathbb{k} \Pi_{n}, \vee\right)$ the operation

$$
\Delta(N)=\sum_{k=0}^{n} \operatorname{Res}_{k, n-k} N
$$

and for $M \in G_{0}\left(\mathbb{k} \Pi_{m}, \vee\right)$,

$$
N \cdot M=\operatorname{Ind}_{n, m} N \otimes M .
$$

$G_{0}(\Pi, \vee)$ with the operation $\Delta$ defines a coalgebra and $G_{0}(\Pi, \vee)$ with the product of (15) defines an algebra structure. It is easily checked that the product and coproduct on $G_{0}(\Pi, \vee)$ are not compatible as a bialgebra structure.

It is interesting to note that $G_{0}(\Pi, \vee)$ endowed with the tensor product (12) and the coproduct $\Delta$ from equation 14 does define a bialgebra. To highlight the relationship with NCSym, we define an internal coproduct $\Delta^{\odot}$ on $K_{0}\left(\mathbb{k}_{k}, \vee \mathrm{V}\right)$ which is the natural dual to equation (12). That is we define a map $\Delta^{\odot}: K_{0}\left(\mathbb{k} \Pi_{n}, \vee\right) \rightarrow K_{0}\left(\mathbb{k} \Pi_{n}, \vee\right) \otimes K_{0}\left(\mathbb{k} \Pi_{n}, \vee\right)$ such that

$$
\Delta^{\odot}\left(W_{A}\right)=\sum_{B \wedge C=A} W_{B} \otimes W_{C} .
$$

We can now show with the following theorem that $K_{0}(\Pi, \vee)$ is a bialgebra and the simple modules in $K_{0}(\Pi, \vee)$ correspond to the $\mathbf{m}$-basis on NCSym.

Theorem 3.5 The ring $K_{0}(\Pi, \vee)$ endowed with product $M \cdot N:=\operatorname{Ind}_{n, m} M \otimes N$ and coproduct $\Delta^{\odot}$ of equation (16) defines a bialgebra. Moreover, the map

$$
F: K_{0}(\Pi, \vee) \rightarrow \text { NCSym }
$$

given by $F\left(W_{A}\right)=\mathbf{m}_{A}$ is an isomorphism of bialgebras.

proof: Recall that NCSym is a bialgebra linearly spanned by elements $\mathbf{m}_{A}$ with the product defined by

$$
\mathbf{m}_{A} \mathbf{m}_{B}=\sum_{C \wedge\left(\mathbf{1}_{n} \mid \mathbf{1}_{m}\right)=A \mid B} \mathbf{m}_{C}
$$

and an inner coproduct defined by

$$
\Delta^{\odot}\left(\mathbf{m}_{A}\right)=\sum_{B \wedge C=A} \mathbf{m}_{B} \otimes \mathbf{m}_{C} .
$$

Equations (16) and (13) show that the map $F\left(W_{A}\right)=\mathbf{m}_{A}$ is an isomorphism of bialgebras. 
This construction that we have presented here in the last two sections of defining an algebra from a lattice operation and looking at the modules is something that can be done in a more general setting and is a tool that can be used to analyze other Hopf algebras. This will be the subject of future work.

\section{Existence of the $\mathrm{x}_{A}$ and Frobenius characteristic}

We now prove our Theorem 1.3. It is useful at this point to introduce an intermediate basis of NCSym. In [17], an analogue of the power sum basis is given by

$$
\mathbf{p}_{A}=\sum_{B \geq A} \mathbf{m}_{B} .
$$

This basis has many nice properties.

Lemma 4.1 The set $\left\{\mathbf{p}_{A}: A \in \Pi_{n}, n \geq 0\right\}$ forms a basis of NCSym such that

(i) $\mathbf{p}_{A} \mathbf{p}_{B}=\mathbf{p}_{A \mid B}$

(ii) $\Delta^{\odot}\left(\mathbf{p}_{A}\right)=\mathbf{p}_{A} \otimes \mathbf{p}_{A}$.

proof: By triangularity, it is clear that the set forms a basis. Now, for $A \in \Pi_{n}$ and $B \in \Pi_{m}$ we have

$$
\mathbf{p}_{A} \mathbf{p}_{B}=\sum_{C \geq A} \sum_{D \geq B} \mathbf{m}_{C} \mathbf{m}_{D}=\sum_{C \geq A} \sum_{D \geq B} \sum_{E \wedge \mathbf{1}_{n}\left|\mathbf{1}_{m}=C\right| D} \mathbf{m}_{E} .
$$

Notice that we have that if $E \wedge \mathbf{1}_{n}\left|\mathbf{1}_{m}=C\right| D$, then $E \geq C|D \geq A| B$. Conversely, if $E \geq A \mid B$, then we find unique $C$ and $D$ such that $C\left|D=E \wedge \mathbf{1}_{n}\right| \mathbf{1}_{m} \geq A\left|B \wedge \mathbf{1}_{n}\right| \mathbf{1}_{m}=$ $\left(A \wedge \mathbf{1}_{n}\right)\left|\left(B \wedge \mathbf{1}_{m}\right)=A\right| B$. This implies that the sum is equal to

$$
\mathbf{p}_{A} \mathbf{p}_{B}=\sum_{E \geq A \mid B} \mathbf{m}_{E}=\mathbf{p}_{A \mid B} .
$$

For the second equality, we have

$$
\begin{aligned}
\Delta^{\odot}\left(\mathbf{p}_{A}\right) & =\sum_{B \geq A} \Delta^{\odot}\left(\mathbf{m}_{B}\right)=\sum_{B \geq A} \sum_{C \wedge D=B} \mathbf{m}_{C} \otimes \mathbf{m}_{D} \\
& =\sum_{C \geq A} \sum_{B \geq A} \sum_{D: C \wedge D=B} \mathbf{m}_{C} \otimes \mathbf{m}_{D}=\sum_{C \geq A} \sum_{D \geq A} \mathbf{m}_{C} \otimes \mathbf{m}_{D} \\
& =\mathbf{p}_{A} \otimes \mathbf{p}_{A} .
\end{aligned}
$$

We finally define our basis. Let

$$
\mathbf{x}_{A}=\sum_{B \leq A} \mu(B, A) \mathbf{p}_{B}
$$

By triangularity, the set $\left\{\mathbf{x}_{A}: A \Pi_{n}, n \geq 0\right\}$ is an integral basis of NCSym. We now see that this basis has the required properties. 


\section{Lemma 4.2}

$$
\begin{aligned}
& \text { (i) } \mathbf{x}_{A} \mathbf{x}_{B}=\mathbf{x}_{A \mid B} \\
& \text { (ii) } \Delta^{\odot}\left(\mathbf{x}_{C}\right)=\sum_{A \vee B=C} \mathbf{x}_{A} \otimes \mathbf{x}_{B} .
\end{aligned}
$$

proof: Using the same argument as in Lemma 2.5 we have

$$
\begin{aligned}
\mathbf{x}_{A} \mathbf{x}_{B} & =\sum_{C \leq A} \sum_{D \leq B} \mu(C, A) \mu(D, B) \mathbf{p}_{C} \mathbf{p}_{D} \\
& =\sum_{C \leq A} \sum_{D \leq B} \mu(C, A) \mu(D, B) \mathbf{p}_{C \mid D}=\sum_{E \leq A \mid B} \mu(E, A \mid B) \mathbf{p}_{E}=\mathbf{x}_{A \mid B} .
\end{aligned}
$$

This shows the first identity. For the second, the left hand side of (ii) is

$$
\Delta^{\odot}\left(\mathbf{x}_{C}\right)=\sum_{E \leq C} \mu(E, C) \Delta^{\odot}\left(\mathbf{p}_{E}\right)=\sum_{E \leq C} \mu(E, C) \mathbf{p}_{E} \otimes \mathbf{p}_{E}
$$

and the right hand side is

$$
\sum_{A \vee B=C} \mathbf{x}_{A} \otimes \mathbf{x}_{B}=\sum_{A \vee B=C} \sum_{\substack{E \leq A \\ F \leq B}} \mu(E, A) \mu(F, B) \mathbf{p}_{E} \otimes \mathbf{p}_{F}
$$

Let us isolate the coefficient of $\mathbf{p}_{E} \otimes \mathbf{p}_{F}$ in the sum above we get

$$
\begin{aligned}
T_{E, F}^{C} & =\sum_{\substack{E \leq A \leq C \\
F \leq B \leq C}} \sum_{A \vee B=C} \mu(E, A) \mu(F, B) \\
& =\sum_{F \leq B \leq C}\left(\sum_{\substack{E \leq A \leq C \\
A \vee B=C}} \mu(E, A)\right) \mu(F, B) .
\end{aligned}
$$

By symmetry (interchanging the role of $E$ and $F$ if needed), we may assume that $F \nless E$. In [19], Corollary 3.9.3 is dual to the following statement

$$
\sum_{\substack{A \leq \mathbf{1}_{n} \\ A \vee B=\mathbf{1}_{n}}} \mu\left(\mathbf{0}_{n}, A\right)= \begin{cases}\mu\left(\mathbf{0}_{n}, \mathbf{1}_{n}\right) & \text { if } B=\mathbf{0}_{n} \\ 0 & \text { otherwise }\end{cases}
$$

where, as usual, $\mathbf{0}_{n}=\{1.2 \ldots n\}$. This implies that the sum of in bracket in equation (20) is equal to

$$
\sum_{\substack{E \leq A \leq C \\ A \vee B=C}} \mu(E, A)= \begin{cases}\mu(E, C) & \text { if } B=E \\ 0 & \text { otherwise }\end{cases}
$$


This follows from the fact that $\mu$ is multiplicative and in general the interval $[E, C] \subseteq \Pi_{n}$ is isomorphic to a cartesian product of (smaller) partition lattices (see Example 3.9.4 in [19]). If we substitute this back in equation (20) we have two cases to consider. When $F \neq E$, our assumption that $F \nless E$ prohibits the possibility that $F \leq B=E$. Thus we must always have $B \neq E$ and in this case $T_{E, F}^{C}=0$. When $F=E$, the only value of $B$ where equation (21) does not vanish is when $B=E=F$ and we get $T_{E, E}^{C}=\mu(E, C) \mu(E, E)=\mu(E, C)$. If we compare this to equation (19) we conclude our proof of (ii).

Notice that the character of the module (the trace of the matrix representing the action of $\left.\mathbb{k} \Pi_{n}\right) V_{B}$ from formula (5) is given by the formula $\chi^{V_{B}}(A)=\delta_{B \leq}(A)$ when $A \in \mathbb{k} \Pi_{n}$ acts on $V_{B}$. We observe that equation (18) for $\mathbf{x}_{A}$ yields

$$
\mathbf{p}_{A}=\sum_{B \leq A} \mathbf{x}_{B}=\sum_{B} \chi^{V_{B}}(A) \mathbf{x}_{B}
$$

This means that the characters for the simple modules for $(\boldsymbol{\Pi}, \wedge)$ are encoded in the change of basis coefficients between the $\mathbf{p}$ and $\mathbf{x}$ basis.

Similarly, the character of the module $W_{B}$ when acted on by the element $A \in \mathbb{k} \prod_{n}$ are given by the formula $\chi^{W_{B}}(A)=\delta_{B \geq}(A)$ from equation (11). Of course the defining relation of the $\mathbf{p}$ basis from equation (17) shows that

$$
\mathbf{p}_{A}=\sum_{B} \chi^{W_{B}}(A) \mathbf{m}_{B}
$$

We observe in this formula that the characters of the simple modules of $(\boldsymbol{\Pi}, \vee)$ are encoded in the change of basis coefficients between the $\mathbf{p}$ and $\mathbf{m}$ basis.

Both these formulas are in fairly close analogy with the formula for the expansion for the power basis in the Schur basis in the algebra of the symmetric functions. There the change of basis coefficients are the characters of the simple modules of the symmetric group. This shows that the p-basis which was defined by Rosas and Sagan [17] does represent the analogue of the power basis in the algebra of the symmetric functions and the $\mathbf{x}$ and the $\mathbf{m}$ bases encode in their coefficients the characters of the modules that they represent.

Remark 4.3 One could also define a third algebra $\left(\mathbb{k} \Pi_{n}\right.$, @) where $A @ B=\delta_{A=B} A$ and construct the simple modules as we have done here for $\left(\mathbb{k}_{n}, \wedge\right)$ and $\left(\mathbb{k} \Pi_{n}, \vee\right)$. This same construction shows that the simple modules of this algebra satisfy a tensor product, induction and restriction operations which make the Grothendieck ring (of the category of the finite dimensional projective modules) for this algebra isomorphic again to NCSym as a bialgebra where the simple modules behave as the elements $\mathbf{p}_{A} \in \mathbf{N C S y m}$ and $\mathbf{p}_{A}$ is defined in (17). 
Remark 4.4 Summary of bases in NCSym.

The $\mathbf{m}$ basis:

$$
\begin{aligned}
\mathbf{m}_{A} \mathbf{m}_{B} & =\sum_{C \wedge\left(\mathbf{1}_{n} \mid \mathbf{1}_{k}\right)=A \mid B} \mathbf{m}_{C} \\
\Delta\left(\mathbf{m}_{A}\right) & =\sum_{S \subseteq[\ell(A)]} \mathbf{m}_{A_{S}} \otimes \mathbf{m}_{A_{S^{c}}} \\
\Delta^{\odot}\left(\mathbf{m}_{A}\right) & =\sum_{B \wedge C=A} \mathbf{m}_{B} \otimes \mathbf{m}_{C}
\end{aligned}
$$

The $\mathbf{p}$ basis:

$$
\begin{aligned}
\mathbf{p}_{A} \mathbf{p}_{B} & =\mathbf{p}_{A \mid B} \\
\Delta\left(\mathbf{p}_{A}\right) & =\sum_{S \subseteq[\ell(A)]} \mathbf{p}_{A_{S}} \otimes \mathbf{p}_{A_{S^{c}}} \\
\Delta^{\odot}\left(\mathbf{p}_{A}\right) & =\mathbf{p}_{A} \otimes \mathbf{p}_{A}
\end{aligned}
$$

The $\mathbf{x}$ basis:

$$
\begin{aligned}
\mathbf{x}_{A} \mathbf{x}_{B} & =\mathbf{x}_{A \mid B} \\
\Delta^{\odot}\left(\mathbf{x}_{A}\right) & =\sum_{B \vee C=A} \mathbf{x}_{B} \otimes \mathbf{x}_{C}
\end{aligned}
$$

It would be interesting to find a formula for $\Delta\left(\mathbf{x}_{A}\right)$.

\section{References}

[1] M. Aguiar, N. Bergeron and F. Sottile, Combinatorial Hopf Algebras and Generalized Dehn-Sommerville Relations, Compositio Mathematica 142 (2006) 1-30.

[2] M. Aguiar And S. Mahajan, Coxeter groups and Hopf algebras, Fields Institute Monographs, Volume 23 (2006), AMS, Providence, RI.

[3] M. Aguiar And F. SotTile, Structure of the Malvenuto-Reutenauer Hopf algebra of permutations, Advances in Mathematics, 191 n2 (2005) 225-275.

[4] P. Baumann And C. Hohlweg, A Solomon's theory for the wreath products $G$ ? $\mathfrak{S}_{n}$, To appear at Transaction of the American Mathematical Society, 64p. ArXiv math.CO/0503011.

[5] N. Bergeron, F. Hivert and J.-Y. Thibon, The peak algebra and the HeckeClifford algebras at $q=0$, Journal of Combinatorial Theory, Series A, 107, 1, (2004), $1-19$.

[6] N. Bergeron, S. Mykytiuk, F. Sottile, and S. Van Willigenburg, Pieri Operations on Posets, Journal of Combinatorial Theory, Series A, 91 (2000), 84-110. 
[7] N. Bergeron, C. Reutenauer, M. Rosas and M. Zabrocki, Invariants and Coinvariants of the Symmetric Group in Noncommuting Variables, to appear at the Canadian Journal of Mathematics, ArXiv math.CO/0502082.

[8] N. Bergeron and M. Zabrocki, The Hopf Algebra of symmetric functions in non-commuting variables is free and cofree, submitted. ArXiv math.CO/0509265.

[9] G. Bergman and P. Cohn, Symmetric elements in free powers of rings, Journal of the London Mathematical Society, 211969 525-534.

[10] K. S. Brown, Semigroups, rings, and Markov chains, Journal of Theoretical Probabability, 13 (2000), no. 3, 871-938.

[11] G. Duchamp, F. Hivert and J. Y. Thibon, Noncommutative symmetric functions. VI. Free quasi-symmetric functions and related algebras, International Journal of Algebra and Computation 12 (2002), no. 5, 671-717.

[12] F. Hivert, J.-C. Novelli And J.-Y. Thibon, Representation theory of the 0Ariki-Koike-Shoji algebras. To appear (2005). ArXiv math.CO/0407218.

[13] M. E. Hoffman, Quasi-shuffle products, Journal of Algebraic Combinatorics, 11 (2000), no. 1, 49-68.

[14] L. Hullan, Thesis, York University, in preparation.

[15] D. Krob and J.-Y. Thibon, Noncommutative symmetric functions IV: Quantum linear groups and Hecke algebras at $q=0$, Journal of Algebraic Combinatorics 6 (1997), 339-376.

[16] I. Macdonald, Symmetric Functions and Hall Polynomials, Oxford Univ. Press, 1995, second edition.

[17] M. Rosas And B. Sagan, Symmetric Functions in Noncommuting Variables, Transactions of the American Mathematical Society, 358 (2006), 215-232.

[18] M. Schocker, The module structure of the Solomon-Tits algebra of the symmetric group, Journal of Algebra 301 (2006), No. 2, 554-586.

[19] R. Stanley, Enumerative Combinatorics, Vol. 1, Cambridge Studies in Advanced Mathematics, 49. Cambridge University Press, Cambridge, 1997.

[20] J. Tits, Two properties of Coxeter complexes. Appendix to "A Mackey formula in the group ring of a Coxeter group" (Journal of Algebra 41 (1976), no. 2, 255-264) by Louis Solomon, Journal of Algebra 41 (1976), no. 2, 265-268.

[21] M. C. Wolf, Symmetric functions of non-commutative elements, Duke Mathematical Journal 2 (1936), 626-637.

[22] A. V. Zelevinsky, Representations of finite classical groups: a Hopf algebra approach, Springer Lecture Notes 869, Springer-Verlag, Berlin-New York (1981). 